Citation:

Michel G (2006). The influence of neuroticism on concurrent symptom reporting: A multilevel modelling approach. Personality and Individual Differences. 41(3), 549-560.

doi:10.1016/j.paid.2006.01.019

\title{
The influence of neuroticism on concurrent symptom reporting: a multilevel modelling approach
}

\author{
Gisela Michel \\ New York University, University of Fribourg, University of Berne
}

\begin{abstract}
Address: Dr. Gisela Michel, Dept of Social and Preventive Medicine, University of Berne, Finkenhubelweg 11, 3012 Berne, Switzerland

Ph: +41316313899

Fax: +41316313520

E-mail: michel@ispm.unibe.ch (new: gisela.michel@unilu.ch)
\end{abstract}

\begin{abstract}
The current study explored the influence of neuroticism on the diurnal pattern of symptom reporting. Participants were 548 individuals from 169 families. Using a computer-assisted selfmonitoring procedure, individuals reported their concurrent somatic symptoms six times per day for seven consecutive days. Neuroticism was assessed separately in a follow-up questionnaire. We used a generalized linear models approach for multilevel-analysis, and analysed a four level model with observations within days, days within individuals, and individuals within families. Results show no main effect for neuroticism, but an interaction of neuroticism with time of the observation. Individuals average in neuroticism show a curvilinear pattern with increased symptom reporting in the morning and in the evening, and less during the day. Individuals with low levels of neuroticism show a similar but more attenuated pattern. Individuals high in neuroticism, however, show a constant level of symptom reporting throughout the day, with no decrease in the middle of the day. These findings suggest that neuroticism results not so much in exaggerated symptom reporting but in a different diurnal pattern of concurrent symptom reporting.
\end{abstract}

Keywords: neuroticism, concurrent symptom reporting, daily pattern, multilevel analysis 


\section{The influence of neuroticism on concurrent symptom reporting: a multilevel modelling approach}

For the last twenty years, neuroticism and negative affectivity have been the most consistent predictors of symptom reporting especially in retrospective questionnaires (Costa \& McCrae, 1980a, 1980b, 1985, 1987; Johnson, 2003; Vassend \& Skrondal, 1999; Watson \& Pennebaker, 1989; Williams \& Wiebe, 2000). In the last decade, a much weaker association has also been shown with concurrent symptom reports (Cohen, Doyle, Skoner, Fireman, \& et al., 1995; Fahrenberg, Bruegner, Foerster, \& Kaeppler, 1999; Feldman, Cohen, Doyle, Skoner, \& Gwaltney, 1999; Kolk, Hanewald, Schagen, \& Gijsbers van Wijk, 2003; Larsen, 1992; Neitzert, Davis, \& Kennedy, 1997; Williams et al., 2002). However, only rarely have the concurrent measures been analysed without aggregating. The current study was designed to examine the influence of neuroticism on concurrent symptom reporting with multiple measures of symptom reporting per day. This procedure allowed an examination of differences between individuals high and low in neuroticism not only on their average level of symptom reporting but in their daily pattern.

\section{Concurrent symptom reporting and its association with neuroticism}

Neuroticism is a stable personality characteristic associated with the experience of negative emotions, such as anxiety, fear, depression and hostility. Often, individuals high in neuroticism are very ruminative, especially regarding their health and well-being. In various questionnaires neuroticism is assessed as emotional instability (in contrast to emotional stability; e.g. Freiburg Personality Inventory, Fahrenberg, Hampel, \& Selg, 2001) or negative affectivity (e.g. the trait measure of the State-Trait Anxiety Inventory, Spielberger, Gorsuch, Lushene, Vagg, \& Jabobs, 1983) or the Positive and Negative Affect Schedule, (Watson, Clark, \& Tellegen, 1988). Because in the published literature the term neuroticism is still widely used as a superordinate concept of emotional stability and negative affectivity, in the current paper the term neuroticism will be used.

In contrast to retrospective studies, prospective studies of symptom reporting show a smaller or no association with neuroticism. A higher report of somatic symptoms is usually found in reports assessed at the end of the day (diary approach; Cohen et al., 1995; Feldman et al., 1999; Kolk et al., 2003; Larsen, 1992). Studies using an experience or time sampling approach on the other hand, find an association between emotional state and symptom reporting but no (Brown \& Moskowitz, 1997), or only a very small association with neuroticism (Fahrenberg et al., 1999; Williams et al., 2002). In diary studies, individuals usually report their symptoms for the past 24 hours. Even if retrospection is abridged in this approach, it is still present to a certain degree. The phenomenon that even in short retrospection, experiences are recalled as worse than they really were, was called retrospection effect (Fahrenberg et al., 1999; Kaeppler \& Rieder, 2001).

These findings suggest that neuroticism only has an effect on health measures if these are recalled from memory; in contrast, emotional states may affect the experience of a somatic event. Nonetheless, high and low neurotic individuals may differ in their somatic experience because of a different prevalence of positive and negative emotional states. While individuals average in neuroticism have an up and down in their emotions during the day (Wilhelm, 2001), high scoring neurotics may experience negative states more frequently, which increases attention to the body, 
makes them more ruminative about somatic experiences, and lets them discover somatic symptoms. Differing daily patterns in neurotics have been found for various psychological characteristics such as emotional states (Fahrenberg et al., 1999; Wilhelm, 2001) and performance indices (Fahrenberg et al., 1999; Kaeppler \& Rieder, 2001), and may also be found in symptom reporting.

\section{Neuroticism and diurnal patterns}

Circadian rhythmicity and its associations with personality characteristics has often been measured using the morningness-eveningness questionnaire (MEQ, Horne \& Östberg, 1976). While some studies did not find differences for neuroticism (e.g. Adan, 1992; Wilson, 1990), others report an association between neuroticism and eveningness (Adan \& Almirall, 1990; Mecacci \& Rocchetti, 1998; Neubauer, 1992), e.g. a higher performance level in the evening, and a peak in body temperature later in the day in high scoring neurotics. These studies, however, rely on a one-time self-evaluation questionnaire to assess variation in diurnal patterns. Studies that compared neuroticism with measures assessed at different times throughout the day are rare. Lund (1974) studied 34 participants who were isolated from any time cues for 20 to 30 days. Whereas most participants showed a synchronized rhythm of activity and body temperature, seven individuals with a high internal desynchronization scored significantly higher in neuroticism. In a more recent study, high scoring neurotics did not show a clear circadian rhythm of positive emotions, and their emotional state was not related to body temperature (Murray, Allen, Trinder, \& Burgess, 2002). The circadian rhythm of body temperature itself was attenuated in highly neurotic individuals. Using an experience sampling method, Rusting and Larsen (1998) found an association between neuroticism and depressive symptomatology in the evening. The preliminary conclusion from these studies can be summarized as a generally weakened circadian rhythmicity in individuals high in neuroticism with the possibility of a worsening of the emotional state in the evening. This may also be the case for the association between neuroticism and symptom reporting. Symptom reporting has been associated with a time pattern of increased complaints in the morning and evening, and decreased complaints during the middle of the day (Goebel \& Cordes, 1990). Whereas this pattern may be true for individuals average in neuroticism, highly neurotic individuals might differ in their circadian pattern.

This study thus investigated if a) there is a difference in the average probability to report symptoms during the day and b) there is a difference in the diurnal pattern of symptom reporting between individuals high and low in neuroticism. We expected no difference in the average probability but a differing diurnal pattern. c) In addition, the structure of the variability in symptom reporting was investigated.

\section{Method}

\section{Participants}

The sample contained families with adolescent children from two separate, but highly similar studies: the Second and the Third Fribourg Family Project. In the Second Fribourg Family Project (Perrez, Schoebi, \& Wilhelm, 2000), 101 families were recruited by sending 
approximately 5200 information letters to families in and around Fribourg, Switzerland. Schools provided addresses of children in $7^{\text {th }}$ grade and higher (13 years and older). In the Third Fribourg Family Project, 78 families were recruited by sending approximately 2600 letters to families. In addition to schools, scout groups from Bern and Fribourg provided help in recruiting, and articles in local and national newspapers and magazines called for participants for a study exploring stress and conflict in families with adolescent children. In the Third Project only families with at least one child aged 13 or 14 years were allowed to participate. Because other research questions addressed the parent-adolescent relationship, only families with two parents and at least one child older than 13 years were allowed to participate (although siblings could participate as well). The requirement that at least three members of a family (including both parents) participated in the study, together with the relatively time-consuming assessment method were mainly responsible for the rather low response rate. It is, however, expected that the sample described did not differ significantly from the general population in the process investigated in the present paper. In the Second Project two out of the 101 families decided not to participate before the self-observation started, two additional families terminated the observation early, and one family experienced many technical problems. Six participants from three families did not return the follow-up questionnaire containing the measure of neuroticism and had to be excluded from the analysis. 308 participants from 95 families were available for the analysis. In the Third Project one family decided not to participate before they started the observation week. Fourteen participants from seven families did not return the follow-up questionnaire leaving 240 individuals from 74 families for the analysis.

Because of highly similar sampling methods as well as no major changes in the self-observation procedure we decided to combine the two samples and control for sample membership in the analysis. The final sample consisted of 548 participants (335 parents and 213 children) in 169 families. The mean age of the parents was 45.2 years $(S D=5.1$; age range $=32$ to 64$)$. The mean age of the children was 14.7 years $(S D=1.3$; age range $=11$ to 19$)$.

\section{Procedure}

After returning an application form, families interested in participating in the project were contacted by phone to clarify any remaining questions and to arrange a date for the selfobservation. In order to introduce all participants thoroughly to the self-observation procedure each family was visited at home by a collaborator of ours who also provided technical support during the observation week (if needed). Each participant (father, mother and at least one child older than 13 years) received a palm computer (HP 200 LX). They started the computer themselves each morning after waking up, and filled in the first questionnaire. During the remainder of the day an alarm sound reminded them five more times to fill in the questionnaire. These signals were randomly distributed within an hour (between 9 and 10am, 12 and 1pm, 3 and 4pm, 6 and 7pm, 9 and 10pm), but at the same time for every family member. 9.2\% of all possible observations were missed. Examples of reasons for missing observations are: being asleep, having an exam at school, having forgotten to fill in the questionnaire before the next observation had started.

Before and after the self-observation procedure, participants filled out questionnaires assessing demographics and other measures (e.g. neuroticism). In the Third Fribourg Family Project, 
another self-observation week followed about three to four months after the first. This second week is not included in the present analysis. Data were gathered between November 1998 and April 2000 (Second Project) and between January 2001 and May 2003 (Third Project).

\section{Measures}

\section{Symptom reporting}

Symptom reporting was measured using a computer assisted self-observation procedure (Perrez et al., 2000). Participants had to answer the question "Do you experience any kind of somatic complaints or pains?”, assessed as a binary response (yes / no; for a description of the distribution of the data see Table 1).

\section{Neuroticism}

The emotionality scale in the Freiburg Personality Inventory (FPI, Fahrenberg et al., 2001) was used to measure neuroticism. The emotionality scale contains 14 items, two of which assess health worries. The scale with the two items omitted was still correlated with the original scale at .98 , so that the original scale was used in the present study. This had the advantage that the raw scores could be recoded into Stanine scores. The internal consistency for of the FPI emotionality scale is alpha $=.80$ (in the current study alpha $=.78$ ), the average inter-item correlation was 0.21 and the range of item-rest correlation was between 0.28 and 5.2. The mean score (in Stanine) in our sample of 548 individuals was $4.32(S D=1.83)$, and therefore slightly lower than in the representative samples described by Fahrenberg et al. (2001) (for mean scores of the family members see Table 2). The Pearson correlation between the raw scores of mothers and fathers was $r=0.105(p=0.180)$, between mothers and first child $r=0.173(p=0.025)$ and between fathers and first child it was $\mathrm{r}=0.148(\mathrm{p}=0.057)$. The two samples differed significantly (Second Project: mean $=4.49 ; S D=1.84$; Third Project: mean $=4.15 ; S D=1.81 ; t=2.161, d f=546 ; p=0.031$, Effect size $=0.185$ ).

\section{Analysis}

In order not to lose any information by aggregating data a generalized linear multilevel approach with a logit link function as implemented in MLwiN (Rasbash et al., 2000) was used (Goldstein, 1995). The present study includes 169 families, with 548 individuals, 3769 days of selfobservation, and 21117 observed occasions. The model analysed is thus a four-level model (with observations within days, within individuals, within families). The multilevel structure does not require balanced data and missing data are thus not a problem for the analysis.

In the current model we include time of the day, neuroticism and the interaction between them as fixed effects. In addition we added a fixed effect for project, to adjust for differences between the two projects. Time was specified as three orthogonal polynomials up to the cubic trend. Polynomials were also used for neuroticism to allow for non-linear increase between different scores. Both the time- and the neuroticism polynomials were centred, such that the intercept represents the probability of symptom reporting for an individual average in neuroticism (Stanine 
$=5$ ) in the middle of the day. For the intercept, random effects on the family, the person and the day level were included. They allow each day within a person and each person within a family, and each family to vary from the other days or individuals or families in their average probability of symptom reporting. Random effects on the same levels were allowed for the three time effects. They allow days, individuals and families not only to have a different general probability of reporting complaints, but also to have different trajectories within days. For neuroticism a random effect on the family level was included to allow for differences between families in their general neuroticism level. On the lowest level, a multiplier was included to allowed for extrabinomial variation (Goldstein, 1995). The following model specification was used:

$$
\begin{aligned}
& \operatorname{Logit}\left(\pi_{\mathrm{ijkl}}\right)=\beta_{1 \mathrm{jkl}}+\beta_{2 \mathrm{jkl}} \mathrm{time} 1_{\mathrm{ijkl}}+\beta_{3 \mathrm{jkl}} \mathrm{time} 2_{\mathrm{ijkl}}+\beta_{4 \mathrm{jkl}} \mathrm{time} 3_{\mathrm{ijkl}} \\
& +\beta_{5 l} \mathrm{~N} 1_{\mathrm{kl}}+\beta_{6} \mathrm{~N} 2_{\mathrm{kl}}+\beta_{7} \mathrm{~N} 3_{\mathrm{kl}} \\
& +\beta_{8} \text { N1.time } 1_{i j k l}+\beta_{9} \text { N2.time } 1_{i j k l}+\beta_{10} \text { N3.time } 1_{i j k l} \\
& +\beta_{11} \text { N1.time } 2_{i j k l}+\beta_{12} \text { N2.time } 2_{i j k l}+\beta_{13} \text { N3.time } 2_{i j k l} \\
& +\beta_{14} \mathrm{~N} \text {.time } 3_{\mathrm{ijkl}}+\beta_{15} \mathrm{~N} 2 . \mathrm{time} 3_{\mathrm{ijkl}}+\beta_{16} \mathrm{~N} . \mathrm{time} 3_{\mathrm{ijkl}} \\
& +\beta_{17} \text { project }_{1} \\
& \beta_{1 \mathrm{jkl}}=\beta_{1}+\mathrm{f}_{1 \mathrm{l}}+\mathrm{v}_{1 \mathrm{kl}}+\mathrm{u}_{1 \mathrm{jkl}} \\
& \beta_{2 j \mathrm{kl}}=\beta_{2}+\mathrm{f}_{2 \mathrm{l}}+\mathrm{v}_{2 \mathrm{kl}}+\mathrm{u}_{2 \mathrm{jkl}} \\
& \beta_{3 \mathrm{jkl}}=\beta_{3}+\mathrm{f}_{3 \mathrm{l}}+\mathrm{v}_{3 \mathrm{kl}}+\mathrm{u}_{3 \mathrm{jkl}} \\
& \beta_{4 \mathrm{jkl}}=\beta_{4}+\mathrm{f}_{4 \mathrm{l}}+\mathrm{v}_{4 \mathrm{kl}}+\mathrm{u}_{4 \mathrm{jkl}} \\
& \beta_{51}=\beta_{5}+f_{51}
\end{aligned}
$$

where $\pi_{\mathrm{ijkl}}$ represents the probability to report a complaint at any occasion $\mathrm{i}$, in day $\mathrm{j}$, in person $\mathrm{k}$, in family $1 . \beta_{1 j \mathrm{kl}}$ represents the intercept, or the log odds to report a complaint in the middle of the day for an individual average in neuroticism. The subscripts jkl show that the intercept is allowed to vary on the day-, the person-, and the family level. $\beta_{1}$ thus represents the fixed effect and $\mathrm{f}_{11}$, $\mathrm{v}_{1 \mathrm{kl}}$, and $\mathrm{u}_{1 \mathrm{jkl}}$ the random effects on the family-, the person-, and the day-level, respectively. This works similarly for the coefficients of time1, time2, and time3, which represent the linear, the quadratic, and the cubic time-effect. Neuroticism is added in a linear, a quadratic and a cubic term as well, symbolized by N1, N2, and N3 (a model with dummy variables for every neuroticism score, and an interaction with the three time effects for each score was analysed as well. The results are highly similar to the model described in the paper. Because the model with a continuous neuroticism measure is more parsimonious, only this model is presented here). Only N1 has a random effect on the family level. This variance is given in $\mathrm{f}_{51}$. Project is a fixed effect added to account for the influence of the membership in one of the two projects. The remaining fixed effects describe the interactions between time-effects and the three neuroticism effects. The estimates in the Tables are shown in log odds, in the Figures they are shown in probabilities.

\section{Results}

For neuroticism, the results show no main effect, i.e. individuals high in neuroticism do not differ significantly from individuals low in neuroticism in their average probability to report symptom (see Table 3, main effects). The three time effects show a positive quadratic and a negative cubic influence. In the morning and in the evening symptom reporting has a higher 
probability (quadratic effect), which is quite stable from late afternoon to evening (cubic effect). The symptom reporting trajectory for the average person can thus be described as a u-shaped curve and corresponds with the trajectory of a person with average neuroticism in Figure 1 (neuroticism score $=5$ ).

The interaction of neuroticism and time indicates a significant difference between these individuals (see Table 3, interaction terms). Individuals low in neuroticism have a low probability of reporting complaints throughout the day. Individuals with average neuroticism scores show a quadratic trend (high probability in the morning which is reduced in the middle of the day and increases again later in the day). For individuals who are very high in neuroticism reporting symptoms increases throughout the day (indicated by the interaction between neuroticism and linear time effect).

Regarding the random effects, the results show that individuals and days within individuals vary significantly (Table 4). There is a significant family-level random effect for neuroticism; families vary significantly in their level of neuroticism, such that individuals in some families are in general lower in their neuroticism scores than individuals in other families. The significant variation on the person- and the day-level indicate that individuals and days within individuals are different in regard to symptom reporting. The extra-binomial variation on the lowest level indicates a significant under-dispersion. Reporting of symptoms within days varies less than would be expected by the binomial distribution.

\section{Discussion}

The present study shows no difference in the average probability of symptom reporting for individuals differing in neuroticism. However, individuals differing in neuroticism tend to differ in their diurnal pattern of symptom reporting. Whereas highly scoring neurotics tend to report symptoms throughout the entire waking day, individuals with average neuroticism scores show a very clear curvilinear pattern. In the morning, symptom reporting is very frequent. In the middle of the day, symptoms are reported less frequently, and in the evening again, an increase in symptom reporting can be observed, which remains more or less stable until later in the night.

Individuals differ from each other in their diurnal pattern, and so do days within individuals (shown in the significant random effects for the time-associated variables). Therefore even if in general individuals report more complaints in the morning and in the evening, each individual has a different trajectory, and this individual trajectory can change from day to day. One person might have problems with getting up in the morning; another might be very exhausted in the evening. Within these people, however, days might have been very different in regard to complaints, e.g. if a cold only started in the second half of the week. We can therefore conclude that despite having a general pattern of symptom reporting, individuals differ in their daily pattern even if neuroticism is taken into account.

The random effects for neuroticism showed that members of one family have different neuroticism scores than members of another family, and members of the same family resemble each other regarding neuroticism. This is in line with twin studies showing that neuroticism, 
similar to the other Big Five Personality traits, is to a large amount heritable (e.g. Loehlin, McCrae, Costa, \& John, 1998), and suggests that there might be some family level variance in symptom reporting, which is due to shared neuroticism levels in the family.

This pattern fits very well with the diurnal pattern of emotional state (Wilhelm, 2001). Individuals normally experience a more positive emotional state during the day and a more negative emotional state in the morning and in the evening. Brown and Moskowitz (1997) found that neuroticism predisposes for more negative mood states, which in turn lead to increased symptom reporting. Another study showed that neuroticism is associated with concurrent symptom reporting, but mediated by negative mood and selective attention (Kolk et al., 2003). Thus, neuroticism especially results in higher symptom reporting during negative mood states as well as in increased selective attention. If individuals high in neuroticism experience less positive states in the middle of the day, and also pay more attention to their body, while others are distracted by other activities (e.g. work, school), this will result in a difference in symptom experience. Additionally, results are in accordance with other studies that found an attenuated circadian rhythm for positive emotional states in highly neurotic individuals (Murray et al., 2002).

\section{Limitations}

Though this study uses a large sample of normally healthy Swiss families with adolescent children, there are only a limited number of individuals with increased neuroticism scores. Only $1.5 \%$ of the 548 participants were very high in neuroticism (Stanine 9 ) and only $13.4 \%$ of the whole sample showed an above average neuroticism score (Stanine 7 to 9). This makes it difficult to get a very clear picture of differences in diurnal rhythms of symptom reporting. In addition, the current sample consists of highly motivated families, where at least three members committed themselves to participate. It is thus not a representative sample of families with adolescents. Nonetheless, the study showed an association between neuroticism and symptom reporting at different times of the day. This should be understood as a first approach to this topic. A future study would profit from particularly including participants high and low in neuroticism. In addition, other variables possibly influencing the association, such as distraction, should be taken into account.

Summing up, this study showed that the effects of neuroticism on symptom reporting may not so much lie in the total amount of symptoms reported, but may influence much more the diurnal pattern of symptom reporting. 


\section{References}

Adan, A. (1992). The influence of age, work schedule and personality on morningness dimension. International Journal of Psychophysiology, 12(2), 95-99.

Adan, A., \& Almirall, H. (1990). Adaptation and standardization of a Spanish version of the morningness-eveningness questionnaire: Individual differences. Personality and Individual Differences, 11(11), 1123-1130.

Brown, K. W., \& Moskowitz, D. S. (1997). Does unhappiness make you sick? The role of affect and neuroticism in the experience of common physical symptoms. Journal of Personality and Social Psychology, 72(4), 907-917.

Cohen, S., Doyle, W. J., Skoner, D. P., Fireman, P., \& et al. (1995). State and trait negative affect as predictors of objective and subjective symptoms of respiratory viral infections. Journal of Personality and Social Psychology, 68(1), 159-169.

Costa, P. T., \& McCrae, R. R. (1980a). Influence of extraversion and neuroticism on subjective well-being: Happy and unhappy people. Journal of Personality and Social Psychology, 38(4), 668-678.

Costa, P. T., \& McCrae, R. R. (1980b). Somatic complaints in males as a function of age and neuroticism: A longitudinal analysis. Journal of Behavioral Medicine, 3(3), 245-257.

Costa, P. T., \& McCrae, R. R. (1985). Hypochondriasis, neuroticism, and aging: When are somatic complaints unfounded? American Psychologist, 40(1), 19-28.

Costa, P. T., \& McCrae, R. R. (1987). Neuroticism, somatic complaints, and disease: Is the bark worse than the bite? Journal of Personality, 55(2), 299-316.

Fahrenberg, J., Bruegner, G., Foerster, F., \& Kaeppler, C. (1999). Ambulatory assessment of diurnal changes with a hand-held computer: Mood, attention and morningnesseveningness. Personality \& Individual Differences, 26(4), 641-656.

Fahrenberg, J., Hampel, R., \& Selg, H. (2001). Das Freiburger Persönlichkeitsinventar. Göttingen: Hogrefe.

Feldman, P. J., Cohen, S., Doyle, W. J., Skoner, D. P., \& Gwaltney, J. M. (1999). The impact of personality on the reporting of unfounded symptoms and illness. Journal of Personality and Social Psychology, 77(2), 370-378.

Goebel, H., \& Cordes, P. (1990). Circadian variation of pain sensitivity in pericranial musculature. Headache, 30(7), 418-422.

Goldstein, H. (1995). Multilevel statistical models. London: Arnold.

Horne, J. A., \& Östberg, O. (1976). A self-assessment questionnaire to determine morningnesseveningness in human circadian rhythms. International Journal of Chronobiology, 4, 97110.

Johnson, M. (2003). The vulnerability status of neuroticism: over-reporting or genuine complaints? Personality \& Individual Differences, 35(4), 877-887.

Kaeppler, C., \& Rieder, S. (2001). Does the retrospection effect hold as a stable phenomenon? First results from a transcultural self-monitoring study of mood and cognitive states in Brazil and Germany. In J. Fahrenberg \& M. Myrtek (Eds.), Progress in Ambulatory Assessment: Computer-assisted psychological and psychophysiological methods in monitoring and field studies (pp. 113-122). Ashland, OH: Hogrefe \& Huber Publishers.

Kolk, A. M., Hanewald, G. J. F. P., Schagen, S., \& Gijsbers van Wijk, C. M. T. (2003). A symptom perception approach to common physical symptoms. Social Science \& 
Medicine, 57(12), 2343-2354.

Larsen, R. J. (1992). Neuroticism and selective encoding and recall of symptoms: evidence from a combined concurrent-retrospective study. Journal of Personality and Social Psychology, 62(3), 480-488.

Loehlin, J. C., McCrae, R. R., Costa, J., Paul T., \& John, O. P. (1998). Heritabilities of Common and Measure-Specific Components of the Big Five Personality Factors,. Journal of Research in Personality, 32(4), 431-453.

Lund, R. (1974). Personality factors and desynchronization of circadian rhythms. Psychosomatic Medicine, 36(3), 224-228.

Mecacci, L., \& Rocchetti, G. (1998). Morning and evening types: stress-related personality aspects. Personality and Individual Differences, 25(3), 537-542.

Murray, G., Allen, N. B., Trinder, J., \& Burgess, H. (2002). Is weakened circadian rhythmicity a characteristic of neuroticism? Journal of Affective Disorders, 72(3), 281-289.

Neitzert, C. S., Davis, C., \& Kennedy, S. H. (1997). Personality factors related to the prevalence of somatic symptoms and medical complaints in a healthy student population. British Journal of Medical Psychology, 70(1), 93-101.

Neubauer, A. C. (1992). Psychometric comparison of two circadian rhythm questionnaires and their relationship with personality. Personality and Individual Differences, 13(2), 125131.

Perrez, M., Schoebi, D., \& Wilhelm, P. (2000). How to assess social regulation of stress and emotions in daily family life? A computer-assisted family self-monitoring system (FASEM-C). Clinical Psychology and Psychotherapy, 7(4), 326-339.

Rasbash, J., Brown, W. J., Goldstein, H., Yang, M., Plewis, I., Healy, M., et al. (2000). A user's guide to MLwiN (second edition). London: Insititute of Education.

Rusting, C. L., \& Larsen, R. J. (1998). Diurnal patterns of unpleasant mood: Associations with neuroticism, depression, and anxiety. Journal of Personality, 66(1), 85-103.

Spielberger, C. D., Gorsuch, R. I., Lushene, R. E., Vagg, P. R., \& Jabobs, G. A. (1983). Manual for the State-Trait Anxiety Inventory (Form Y) (self-evaluation questionnaire). Palo Alto (CA): Consulting Psychologist Press, Inc.

Vassend, O., \& Skrondal, A. (1999). The role of negative affectivity in self-assessment of health: A structural equation approach. Journal of Health Psychology, 4(4), 465-482.

Watson, D., Clark, L. A., \& Tellegen, A. (1988). Development and validation of brief measurs of Positive and Negative Affect: The PANAS Scale. Journal of Personality and Social Psychology, 54, 1063-1070.

Watson, D., \& Pennebaker, J. W. (1989). Health Complaints, Stress, and Distress: Exploring the Central Role of Negative Affectivity. Psychological Review, 96(2), 234-254.

Wilhelm, P. (2001). A multilevel approach to analyze ambulatory assessment data: An examination of family members' emotional states in daily life. In J. Fahrenberg \& M. Myrtek (Eds.), Progress in Ambulatory Assessment: Computer-assisted psychological and psychophysiological methods in monitoring and field studies (pp. 173-189). Kirkland, WA: Hogrefe \& Huber Publishers.

Williams, P. G., Colder, C. R., Lane, J. D., McCaskill, C. C., Feinglos, M. N., \& Surwit, R. S. (2002). Examination of the neuroticism-symptom reporting relationship in individuals with type-2 diabetes. Personality \& Social Psychology Bulletin, 28(8), 1015-1025.

Williams, P. G., \& Wiebe, D. J. (2000). Individual differences in self-assessed health: Gender, neuroticism and physical symptom reports. Personality and Individual Differences, 28(5), 
823-835.

Wilson, G. D. (1990). Personality, time of day and arousal. Personality and Individual Differences, 11(2), 153-168. 


\section{Author Note}

This research was supported by the Swiss National Science Foundation Grant 5004-047773 and 5004-47770 and by a Scholarship from the Swiss National Science Foundation (81FR-068850). The Fribourg Family Projects were initiated by Meinrad Perrez. I would like to thank Niall Bolger for his helpful comments on earlier versions of the draft. 
Table 1: Distribution of complaints during the day for parents and children

\begin{tabular}{|c|c|c|c|c|c|c|}
\hline \multirow[b]{2}{*}{$\begin{array}{l}\text { Complaints reported } \\
\text { Time of day }\end{array}$} & \multicolumn{2}{|c|}{$\begin{array}{c}\text { All participants } \\
\text { (Total observations } \\
\text { N=21117) }\end{array}$} & \multicolumn{2}{|c|}{$\begin{array}{l}\text { Parents (Total } \\
\text { observations } \\
\mathrm{N}=13095 \text { ) }\end{array}$} & \multicolumn{2}{|c|}{$\begin{array}{c}\text { Children (Total } \\
\text { observations } \\
\mathrm{N}=8022)\end{array}$} \\
\hline & Yes & (\%) & Yes & $(\%)$ & Yes & $(\%)$ \\
\hline Getting up & 761 & (21.9) & 493 & (22.8) & 268 & (20.5) \\
\hline Morning & 602 & $(17.2)$ & 377 & (17.3) & 225 & (16.9) \\
\hline Lunch & 613 & (17.3) & 343 & (15.6) & 270 & (20.0) \\
\hline Afternoon & 611 & (17.3) & 356 & (16.2) & 255 & (18.9) \\
\hline Late afternoon & 698 & (19.4) & 376 & (17.0) & 322 & $(23.2)$ \\
\hline Evening & 671 & (19.4) & 356 & (16.5) & 315 & $(24.2)$ \\
\hline Total & 3956 & $(18.7)$ & 2301 & $(17.6)$ & 1655 & $(20.6)$ \\
\hline
\end{tabular}

Table 2: Descriptive statistics of emotionality raw scores of the Freiburg Personality Inventory in family members

\begin{tabular}{lcr}
\hline & $\mathrm{N}$ & Mean Score(SD) \\
\hline Parents & & \\
$\quad$ Males & 167 & $4.63(3.27)$ \\
Females & 168 & $5.43(3.37)$ \\
Children & & \\
Males & 106 & $5.05(3.11)$ \\
Females & 107 & $5.44(3.14)$ \\
\hline
\end{tabular}


Table 3: Estimates and SE of fixed effects for the main effects and interactions of time and neuroticism (in log odds)

\begin{tabular}{lcccc}
\hline & Estimate & $S E$ & $\chi^{2}$ & $p$ \\
\hline Main effects & & & & \\
Intercept & -1.422 & 0.103 & 189.731 & $<0.001$ \\
Time1 & -0.010 & 0.007 & 1.735 & 0.186 \\
Time2 & 0.035 & 0.006 & 36.251 & $<0.001$ \\
Time3 & -0.015 & 0.003 & 22.163 & $<0.001$ \\
N linear & 0.096 & 0.069 & 1.895 & 0.169 \\
N quadratic & 0.008 & 0.018 & 0.220 & 0.639 \\
N cubic & 0.002 & 0.007 & 0.045 & 0.832 \\
Project & -0.066 & 0.128 & 0.263 & 0.608 \\
Interaction terms & & & & \\
N lineartime1 & -0.011 & 0.005 & 4.246 & 0.039 \\
N linear.x.time2 & 0.007 & 0.004 & 2.549 & 0.110 \\
N linear.x.time3 & -0.001 & 0.002 & 0.340 & 0.560 \\
N quadratic.x.time1 & 0.001 & 0.001 & 0.230 & 0.632 \\
N quadratic.x.time2 & -0.002 & 0.001 & 2.156 & 0.142 \\
N quadratic.x.time3 & 0.001 & 0.001 & 3.191 & 0.074 \\
N cubic.x.time1 & 0.001 & 0.001 & 3.336 & 0.068 \\
N cubic.x.time2 & -0.001 & 0.000 & 2.189 & 0.139 \\
N cubic.x.time3 & 0.000 & 0.000 & 0.054 & 0.816 \\
\hline Not N Neurotism
\end{tabular}

Note: N: Neuroticism, time1: linear time effect, time2: quadratic time effect, time3: cubic time effect, project: effect of project (Project 3 vs. Project 2) 
Table 4: Estimates and SE of the random effects for time and neuroticism (in log odds)

\begin{tabular}{cllll}
\hline & Estimate & SE & $\chi 2$ & $\mathrm{p}$ \\
\hline Family Level: Variances & & & & \\
intercept/intercept & 0.000 & 0.000 & n.a. & \\
time1/time1 & 0.001 & 0.001 & 3.796 & 0.051 \\
time2/time2 & 0.000 & 0.000 & n.a. & \\
time3/time3 & 0.000 & 0.000 & n.a. & \\
N_linear/N_linear & 0.056 & 0.025 & 5.042 & 0.025 \\
\hline Person Level: Variances & & & & \\
intercept/intercept & 1.794 & 0.143 & 156.812 & $<0.001$ \\
time1/time1 & 0.003 & 0.001 & 11.912 & $<0.001$ \\
time2/time2 & 0.003 & 0.001 & 22.607 & $<0.001$ \\
time3/time3 & 0.000 & 0.000 & 2.732 & 0.098 \\
\hline Day Level: Variances & & & & \\
intercept/intercept & 1.354 & 0.047 & 829.304 & $<0.001$ \\
time1/time1 & 0.027 & 0.002 & 224.702 & $<0.001$ \\
time2/time2 & 0.012 & 0.001 & 89.135 & $<0.001$ \\
time3/time3 & 0.003 & 0.001 & 27.812 & $<0.001$ \\
\hline Extra-binomial Multiplier & 0.405 & 0.007 & 3457.286 & $<0.001$ \\
\hline -2*Log Likelihood & 10630.1 & & & \\
\hline
\end{tabular}

Note: N: Neuroticism, time1: linear time effect, time2: quadratic time effect, time3: cubic time effect; Covariances are not shown but can be obtained from the author. 
Figure 1: Estimated probabilities of symptom reporting during the day for different neuroticism scores

Note: The estimated probability describes the probability of an individual to answer yes at a certain point in time when asked about having complaints



